\title{
ON CERTAIN QUASI-COMPLEMENTED AND COMPLEMENTED BANACH ALGEBRAS
}

\author{
PAK-KEN WONG \\ Department of Mathematics \\ Seton Hall University \\ South Orange, New Jersey 07079 U.S.A.
}

(Received February 27, 1978)

ABSTRACT. In this paper, we continue the study of quasi-complemented algebras and complemented algebras. The former are generalizations of the latter and were introduced in [4] and studied in [4] and [11]. Some results are proved.

KEY WORDS AND PHRASES. Quasi-complemented and complemented Banach algebras. AMS (MOS) SUBJECT CLASSIFICATION (1970) CODES.

1. INTRODUCTION.

Quasi-complemented algebras, which are generalizations of complemented algebras, were introduced in [4] and studied in [4] and [11]. In this paper, we continue the study of these two classes of algebras.

In Section 3, we introduce the concept of continuous quasi-complementor on a semi-simple annihilator Banach algebra. This is similar to the concept 
of continuous complementor given by Alexander in [1]. Let $A$ be a simple annihilator Banach algebra such that $x \in c 1_{A}(x A)$ for all $x$ in $A$. If $A$ is infinite dimensional, we show that every quasi-complementor on $A$ is continuous. This result is not true if $A$ is finite dimensional. In this case, we obtain that a quasi-complementor $q$ on $A$ is continuous if and only if the set $E_{q}$ of all q-projections is closed and bounded in $A$. By using these results, we give a characterization of continuous quasi-complementors (Theorem $3.4)$.

Section 4 is devoted to the study of uniformly continuous quasi-complementors. Let $A$ be a semi-simple annihilator Banach algebra in which $x \in c 1_{A}(x A)$ for all $x$ in $A$ and $q$ a quasi-complementor on $A$. Suppose that $A$ has no minimal left ideals of dimension less than three. Then we show that $A$ is a dense subalgebra of some dual $B *$-algebra $B$ and $R^{q}=\ell(R) * \cap A$ for all closed right ideals $R$ of $A$. Also every continuous complementor on $A$ is uniformly continuous.

\section{NOTATION AND PRELIMINARIES.}

For any subset $S$ in an algebra $A$, let $\ell_{A}(S)$ and $r_{A}(S)$ denote the left and right annihilators of $S$ in $A$, respectively. Let $A$ be a Banach algebra. Then $A$ is called an annihilator algebra, if for every closed left ideal $J$ and for every closed right ideal $R$, we have $r_{A}(J)=(0)$ if and only if $J=A$ and $\ell_{A}(R)=(0)$ if and only if $R=A$. If $\ell_{A}\left(r_{A}(J)\right)=J$ and $r_{A}\left(l_{A}(R)\right)=R$, then $A$ is called a dual algebra.

Let $A$ be a Banach algebra which is a subalgebra of a Banach algebra $B$. For each subset $S$ of $A, c 1(S)$ (resp. $c 1_{A}(S)$ ) will denote the closure of $S$ in $B$ (resp. $A$ ). Also $\ell(S)$ and $r(S)$ (resp. $\ell_{A}(S)$ and $r_{A}(S)$ ) denote the left and right annihilators of $S$ in $B$ (resp. A). We write $\|\cdot\|$ for the norm on $A$ and $|\cdot|$ for the norm on $B$. 
Let $A$ be a Banach algebra and let $L_{r}$ be the set of all closed right ideals in A. Following [4], we shall say that $A$ is a (right) quasi-complemented algebra if there exists a mapping $q: R \rightarrow R^{q}$ of $L_{r}$ into itself having the following properties:

$$
\begin{array}{ll}
\mathrm{R} \cap \mathrm{R}^{\mathrm{q}}=(0) & \left(\mathrm{R} \varepsilon \mathrm{L}_{\mathrm{r}}\right) ; \\
\left(\mathrm{R}^{\mathrm{q}}\right)^{\mathrm{q}}=\mathrm{R} & \left(\mathrm{R} \varepsilon \mathrm{L}_{\mathrm{r}}\right) ; \\
\text { if } \mathrm{R}_{1} \supset \mathrm{R}_{2}, \text { then } & \mathrm{R}_{2}^{\mathrm{q}} \supset \mathrm{R}_{1}^{\mathrm{q}} \quad\left(\mathrm{R}_{1}, \mathrm{R}_{2} \varepsilon \mathrm{L}_{\mathrm{r}}\right) .
\end{array}
$$

The mapping $\mathrm{q}$ is called a (right) quasi-complementor on A. We know that $R+R^{q}$ is always dense in $A, A^{q}=(0)$ and $(0)^{q}=A$ (see [4]). Hence $R^{q}=(0)$ if and only if $R=A$.

A quasi-complemented algebra $\mathrm{A}$ is called a (right) complemented algebra if it satisfies:

$$
R+R^{q}=A \quad\left(R \in L_{r}\right) .
$$

In this case, the mapping $\mathrm{q}$ is called a ( $\mathrm{right}$ ) complementor on $\mathrm{A}$ (see [6, p. 651, Definition 1]).

Let $A$ be a semi-simple Banach algebra with a quasi-complementor $q$. A minimal idempotent $f$ in $A$ is called a q-projection if $(f A)^{q}=(1-f) A$. The set of all q-projection in $A$ is denoted by $E_{q^{*}}$ By Lemma 3.1 in [11], every non-zero right ideal of A contains a q-projection.

In this paper, all algebras and linear spaces under consideration are over the complex field. Definitions not explicitly given are taken from Rickart's book [5].

We end the section with two new examples of complemented and quasi-complemented algebras.

EXAMPLE 1. Let $A$ be a dual $B *$-algebra and $\Phi$ a symmetric norming function. Then the algebra $A_{\Phi}^{(0)}$ given in $[10, p .293]$ is a complemented algebra with the complementor $q: R \rightarrow \ell_{\Phi}^{(0)}(R) *$ (Theorem 3.4 in [11]). 
EXAMPLE 2. Let $G$ be an infinite compact group with the Haar measure and $A$ the algebra of all continuous functions on $G$, normed by the maximum of the absolute value and $\mathrm{L}_{1}(G)$ the group algebra. It is well known that $A$ and $\mathrm{L}_{1}(\mathrm{G})$ are dual $\mathrm{A}^{*}-\mathrm{algebras}$ which are not two-sided ideals of their completions in an auxiliary norm. It is easy to see that the mapping $q: R \rightarrow l_{A}(R) *$ (resp. $R \rightarrow \ell_{L_{1}(G)}(R) *$ ) is a quasi-complementor on $A$ (resp. $L_{1}(G)$ ). However, by Theorem 3.4 in [11], $\mathrm{q}$ is not a complementor.

\section{CONTINUOUS QUASI-COMPLEMENTORS.}

Let $\mathrm{A}$ be a semi-simple annihflator Banach algebra with a quasi-complementor $q$ and $M_{A}$ the set of all minimal right ideals of $A$. For each $R \varepsilon M_{A}$, by Lemma 3.1 in [11], $R=f A$ for some $q$-projection $f$ in A. Therefore, $R+R^{q}=f A+(1-f) A$. Let $P_{R}$ be the projection on $R$ along $R^{q}$. Then $P_{R}$ is continuous.

DEFINITION. Suppose $a_{n} \varepsilon A$ with $a_{n} A \in M_{A}(n=0,1,2, \ldots)$. A quasicomplementor $\mathrm{q}$ on $\mathrm{A}$ is said to be continuous if whenever $a_{n}$ converges to $a_{0}$, then $\mathrm{P}_{\mathrm{a}_{\mathrm{n}} \mathrm{A}}$ converges to $\mathrm{P}_{\mathrm{a}_{0} \mathrm{~A}}$ uniformly on any minimal left ideal of $\mathrm{A}$.

REMARK. This is similar to the definition of continuous complementor introduced by Alexander (see [1, p. 387, Definition]).

Let $\mathrm{A}$ be a semi-simple annihilator quasi-complemented Banach algebra such that $x \in c l_{A}(x A)$ for all $x$ in $A$ and $\left\{I_{\lambda}: \lambda \varepsilon \Lambda\right\}$ the family of al1 minimal closed two-sided ideals of $A$. Define $q_{\lambda}$ by ${ }^{\mathrm{q}}{ }^{\lambda}=\mathrm{R}^{\mathrm{q}} \bigcap \mathrm{I}_{\lambda}$ for all closed right ideals $R$ of $I_{\lambda}$. Then by [4, p. 144, Theorem 3.6] A is the direct topological sum of $\left\{I_{\lambda}: \lambda \varepsilon \Lambda\right\}$ and $q_{\lambda}$ is a quasi-complementor on $I_{\lambda}$. Let $H_{\lambda}$ be a minimal left ideal of $I_{\lambda}$. Then $H_{\lambda}$ is a Hilbert space under some equivalent inner product norm by [4, p. 145, Lemma 4.2] Let $B_{\lambda}$ be the algebra of all completely continuous linear operators on $H_{\lambda}$. 
Then by the proof of [4, p. 146, Theorem 4.3], $I_{\lambda}$ is a dense subalgebra of B such that $\|\cdot\|$ majorizes $|\cdot|$ on $I_{\lambda}$. By the proof of [8, p. 442, Lemma 5.1], $\mathrm{B}_{\lambda}$ and $\mathrm{I}_{\lambda}$ have the same socle.

LEMMA 3.1. A quasi-complementor $\mathrm{q}$ on $\mathrm{A}$ is continuous if and only if each $q_{\lambda}$ is continuous.

PROOF. Let $R \in M_{A}$ with $R \in I_{\lambda_{0}}$ for some $\lambda_{0} \varepsilon \Lambda$. Then $R=f A$, where $f$ is a q-projection in $I_{\lambda_{0}}$. Hence, for all $x$ in $A, P_{R}(x)=f x$. If $\lambda \neq \lambda_{0}$, then $I_{\lambda_{0}} I_{\lambda}=(0)$ and so $P_{R}(x)=0$ for all $x$ in $I_{\lambda}$. Using this fact and the proof of $[1, \mathrm{p} .387$, Theorem 2.2], we can show that $q$ is continuous if and only if each $\mathrm{q}_{\lambda}$ is continuous.

The following result is a generalization of [3, p. 471, Theorem 6.8].

LEMMA 3.2. Let $A$ be a simple annihilator Banach algebra in which $x \in c 1_{A}(x A)$ for $a 11 \quad x$ in $A$. If $A$ is infinite dimensional, then every quasi-complementor $\mathrm{q}$ on $\mathrm{A}$ is continuous.

PROOF. Let $H$ be a minimal left ideal of $A$. As observed before, $H$ is a Hilbert space under some equivalent inner product and $A$ is a dense dual subalgebra of $B$, the algebra of all completely continuous linear operators on $H$. Also ||$\cdot||$ majorizes $|\cdot|$ on $A$ and $H$ is a minimal left ideal of $B$. Then by $[4, \mathrm{p} .148$, Theorem 5.4], $\mathrm{q}$ can be extended to a quasi-complementor $p$ on $\mathrm{B} ; \mathrm{M}^{\mathrm{p}}=\mathrm{cl}\left([\mathrm{M} \cap \mathrm{A}]^{\mathrm{q}}\right)$ for all closed right ideals $\mathrm{M}$ of $\mathrm{B}$. We show that $M^{P}=\ell(M) *$. In fact, let $S(M)$ be the smallest closed subspace of $H$ that contains the range $x(H)$ for $a 11 \quad x$ in $M$. Since ||$\cdot||$ and $|\cdot|$ are equivalent on $\mathrm{H}$, it follows from [4, p. 145, Lemma 4.1] that

$$
S(M)=M \cap H=M H=(M \cap A) \cap H=(M \cap A) H .
$$

Therefore, we have

$$
S\left(M^{p}\right)=M^{P} H=c l\left([M \cap A]^{q}\right) \cap H=[M \cap A]^{q} \cap H .
$$


(see [4, p. 148] for the last equality). By the proof of [4, p. 145, Lemma 4.2], $M \cap A={ }^{c 1} A((M A) H A)$. Since $A$ is infinite dimensional, by [4, p. 145, Theorem 4.2 (iii)] and (3.1)

$$
\begin{aligned}
S(M)^{\perp} & \left.=\left[{ }^{\perp} 1_{A}(S(M) A)\right]^{q} \cap H=\left[{ }^{H} 1_{A}((M \cap A) H A)\right)\right]^{q} \cap H \\
& =[M \cap A]^{\mathrm{q}} \cap H .
\end{aligned}
$$

Therefore, by (3.2), $\mathrm{S}(\mathrm{M}))^{\perp}=\mathrm{S}\left(\mathrm{M}^{\mathrm{p}}\right)$. Hence it follows from [3, p. 464, Lemma 4.1] and [3, p. 465, Theorem 4.2] that $M^{P}=\ell(M) *$. In particular, $p$ is continuous by $[1, \mathrm{p} .388$, Theorem 2.4].

$$
\text { Suppose } a_{n} A \in M_{A}(n=0,1,2, \ldots) \text { with } a_{n} \rightarrow a_{0} \text { in }\|\cdot\| \text {. }
$$

Hence $a_{n} \rightarrow a_{0}$ in $|\cdot|$. Let $L$ be a minimal left ideal of $A$. Then $L$ is a minimal left ideal of $B$ and $\|\cdot\|$ and $|\cdot|$ are equivalent on $L$; also $a_{n} A=a_{n} B$ for all $n$. Let $f_{n}$ be a (unique) q-projection contained in $a_{n} A$. Then $P_{a_{n} A}(x)=f_{n} x$ for $a l l \quad x$ in $A$. Since $p$ is continuous, $P_{a_{n} A}$ converges to $\mathrm{P}_{\mathrm{a}_{0} \mathrm{~A}}$ uniformly on $\mathrm{L}$ in $|\cdot|$ and hence in $\|\cdot\|$. Therefore $q$ is continuous and this completes the proof.

Let $A$ be a semi-simple annihilator quasi-complemented Banach algebra such that $x \in c l_{A}(x A)$ for all $x$ in $A$ which is a dense subalgebra of a $B^{*}$-algebra B. Suppose $\|\cdot\|$ majorizes $|\cdot|$ on A. By $[8, p .442$, Lemma 5.1], the set $E$ of all hermitian minimal idempotents of $B$ is contained in the socle of $A$ and so $E \subset A$. Let $E_{q}$ be the set of all q-projections in A. For each e $\varepsilon E$, by [4, p. 149, Lemma 6.4], there exists a unique element $Q(e) \varepsilon E_{q}$ such that $Q(e) A=e A$; the mapping $Q: e \rightarrow Q(e)$ is a one - one mapping from $E$ onto $E_{q}$ and is called the q-derived mapping (see [3] and [4]).

As shown in [3, p. 475], Lemma 3.2 is not true in general, if the algebra $A$ is finite dimensional. In this case, we have the following result: 
LEMMA 3.3. Let $A$ be a simple finite dimensional annihilator Banach algebra with a quasi-complementor $\mathrm{q}$ and $\mathrm{E}_{\mathrm{q}}$ the set of all q-projections In $A$. Then $q$ is continuous if and only if $E_{q}$ is a closed and bounded subset of A.

PROOF. By [4, p. 143, Corollany 3.2], $\mathrm{q}$ is a complementor on A. Let $\mathrm{H}$ be a minimal left ideal of $A$. Then $H$ is a Hilbert space and $A$ can be taken as the $\mathrm{B}^{*}-$ algebra of all linear operators on $\mathrm{H}$. Let $\mathrm{Q}$ be the q-derived mapping. By [1, p. 388, Theorem 2.4], Q is continuous if and only if $q$ is continuous. Now Lemma 3.3 follows from Lemma 4.1 in [11].

We have the main result of this section.

THEOREM 3.4. Let A be a semi-simple annihilator quasi-complemented Banach algebra such that $x \in c l_{A}(x A)$ for all $x$ in $A$ and let $\Lambda_{0}=\left\{\lambda \varepsilon \Lambda: I_{\lambda}\right.$ is finite dimensional $\}$. Then a quasi-complementor $q$ on $A$ is continuous if and only if $\mathrm{E}_{\mathrm{q}}^{\lambda}$ is closed and bounded for each $\lambda \varepsilon \Lambda_{0}$, where $\mathrm{E}_{\mathrm{q}}^{\lambda}$ is the set of all q-projections in $\mathrm{I}_{\lambda}$.

PROOF. This follows from Lemma $3.1,3.2$ and 3.3 .

\section{UNIFORMLY CONTINUOUS QUASI-COMPLEMENTORS.}

In this section, we assume that $A$ is a semi-simple annihilator Banach algebra with a quasi-complementor $q$ such that $x \in c 1_{A}(x A)$ for all $x$ in A. Once again, $M_{A}$ will be the set of all minimal right ideals of $A$ and $E_{q}$ the set of all q-projections in $A$. Also let $I_{\lambda}, H_{\lambda}, q_{\lambda}$ and $B_{\lambda}$ be as in §3. The norm on $B_{\lambda}$ is denoted by $|\cdot|$.

DEFINITION. A quasi-complementor $\mathrm{q}$ on $\mathrm{A}$ is said to be uniformly continuous if $\left\{\mathrm{P}_{\mathrm{fA}}: \mathrm{f} \in \mathrm{E}_{\mathrm{q}}\right\}$ is closed and bounded with respect to $\left\|\mathrm{P}_{\mathrm{fA}}\right\|$, the operator bound norm of $\mathrm{P}_{\mathrm{fA}}$.

REMARK. A uniformly continuous quasi-complementor $q$ is continuous. In fact, by Theorem 3.4, we can assume that $A$ is simple and finite dimensional. 
Let $\mathrm{H}$ be a minimal left ideal of $A$. By the proof of Lemma 3.3, A can be taken as the $B^{*}$-algebra of all linear operators on $H$. Then by $[7$, p. 259, Theorem 4], $\mathrm{E}_{\mathrm{q}}$ is bounded. Since $\|\mathrm{f}\|=\sup \{|| \mathrm{fh}||: h \varepsilon H$ and ||$h|| \leq 1\}$, we have $\left\|\mathrm{P}_{\mathrm{fA}}\right\|=\|\mathrm{f}\|$ for all $\mathrm{f} \varepsilon \mathrm{E}_{\mathrm{q}}$. It follows now that $\mathrm{E}_{\mathrm{q}}$ is closed. Hence by Theorem 3.4, $\mathrm{q}$ is continuous.

If $\mathrm{u}$ and $\mathrm{v}$ are elements of a Hilbert space $\mathrm{H}, \mathrm{u} \otimes \mathrm{v}$ will denote the operator on $H$ defined by the relation $(U \otimes v)(h)=(h, v) u$ for all $h$ in $H$. THEOREM 4.1. Let $A$ be a semi-simple annihilator Banach algebra with a uniformly continuous quasi-complementor $q$ in which $x \in c 1_{A}(x A)$ for all $x$ in A. Suppose that $A$ has no minimal left ideals of dimension less than three. Then $A$ is a dense subalgebra of some dual $B^{*}-a l g e b r a ~ B$ and $\mathrm{R}^{\mathrm{q}}=\ell(\mathrm{R}) * \cap \mathrm{A}$ for all closed right ideals $\mathrm{R}$ of $\mathrm{A}$.

PROOF. We know that $\mathrm{q}$ is continuous and so is $\mathrm{q}_{\lambda}(\lambda \varepsilon \Lambda)$. By [4, p. 148, Theorem 5.4], $q_{\lambda}$ induces a quasi-complementor $\mathrm{p}_{\lambda}$ on $\mathrm{B}_{\lambda}$. If $\mathrm{H}_{\lambda}$ is finite dimensional, then by $\left[4, p .143\right.$, Corollary 3.2], $q_{\lambda}$ is a complementor and so by the proof of Theorem 4.3 in [11], $p_{\lambda}$ has the form $J_{\lambda}^{p_{\lambda}}=\ell\left(J_{\lambda}\right)$ * for all closed right ideals $J_{\lambda}$ in $B_{\lambda}$. If $H_{\lambda}$ is infinite dimensional, this is also true by the proof of Lemma 3.2 .

We show that there exists a constant $M$ such that

$$
|| \mathrm{h}|| \leq|\mathrm{h}| \leq \mathrm{M}|| \mathrm{h}|| \quad\left(\mathrm{h} \varepsilon \mathrm{H}_{\lambda}, \lambda \varepsilon \Lambda\right) \text {. }
$$

We follow the argument in [1, p. 393, Lemma 4.3]. It can be assumed that

$$
|| h|| \leq|h| \pm \sqrt{2}|| h|| \quad\left(h \varepsilon H_{\lambda}, \lambda \varepsilon \Lambda\right) \text {. }
$$

Suppose (4.1) does not hold. Then there exists $x_{n}$ in $H_{n}$ such that ||$x_{n}||=1$ and $\left|x_{n}\right|=k_{n}>n$. By (4.2), we can find $z_{n}$ in $H_{n}$ such that ||$z_{n}||=1,\left|z_{n}\right| \leq \sqrt{2}$. Write $z_{n}=\alpha_{n} x_{n}+x_{n}^{\prime}$ with $\alpha_{n} \varepsilon C, x_{n}^{\prime} \varepsilon H_{n}$ and $\left(x_{n}, x_{n}^{\prime}\right)=0$. Put $y_{n}=k_{n}^{-1} x_{n}+x_{n}^{\prime}$ and $f_{n}=\left(y_{n} \otimes y_{n}\right) /\left(y_{n}, y_{n}\right)$. Then $\mathrm{f}_{\mathrm{n}} \in \mathrm{E}_{\mathrm{q}}$ and 


$$
\left\|P_{f_{n} A}\left(x_{n}\right)\right\|=|| \frac{y_{n} \otimes y_{n}}{\left(y_{n}, y_{n}\right) x_{n}}\left\|=\frac{\left|\left(x_{n}, y_{n}\right)\right|}{\left(y_{n}, y_{n}\right)}|| y_{n}\right\| \rightarrow \infty \text {. }
$$

Hence $\left\{\| P_{f_{n}} A \mid\right\}$ is unbounded and this contradicts the uniform continuity of q. Therefore (4.1) holds. Now by using the argument in Theorem 4.3, in [11], we can complete the proof.

Theorem 4.1 shows that there is essentially one type of uniformly continuous quasi-complementors on A.

The following result generalizes [4, p. 153, Theorem 7.6].

COROLLARY 4.2. Let $A$ and $B$ be as in Theorem 4.1. Then $q$ is a complementor on $A$ if and only if $A$ is a left ideal of $B$.

PROOF. This follows from Theorem 4.1 and Theorem 3.4 in [11].

On the other hand, if $\mathrm{q}$ is a complementor, then we have:

THEOREM 4.3. Let $A$ be a semi-simple annihilator Banach algebra such that A has no minimal left ideal of dimension less than three. Then every continuous complementor $\mathrm{q}$ on $\mathrm{A}$ is uniformly continuous.

PROOF. By [6, p. 655, Theorem 4], A is the direct topological sum of its minimal closed two-sided ideals $\left\{I_{\lambda}: \lambda \varepsilon \Lambda\right\}$ each of which is a complemented and dual algebra. Let $\mathrm{q}_{\lambda}, \mathrm{H}_{\lambda}$ and $\mathrm{B}_{\lambda}$ be as before and $|\cdot|$ the norm on $\mathrm{B}_{\lambda}$. By $\left[1, \mathrm{p} .390\right.$, Theorem 3.2], $\mathrm{q}_{\lambda}$ induces a complementor $\mathrm{p}_{\lambda}$ on $\mathrm{B}_{\lambda}$ and by [1, p. 391, Theorem 3.3], $\mathrm{p}_{\lambda}$ has the form $\mathrm{J}_{\lambda}^{\mathrm{p}_{\lambda}}=\ell\left(\mathrm{J}_{\lambda}\right)$ * for all closed right ideals $J_{\lambda}$ in $B_{\lambda}$. By $[1, p .393$, Lemma 4.3], there exists a constant $M$ such that

$$
|| \mathrm{h}|| \leq|\mathrm{h}| \leq \mathrm{M}|| \mathrm{h}|| \quad\left(\mathrm{h} \in \mathrm{H}_{\lambda}, \lambda \in \Lambda\right) .
$$

Let $B$ be the $B^{*}(\infty)$-sum of $\left\{B_{\lambda}: \lambda \varepsilon \Lambda\right\}$. Then $B$ is a dual $B^{*}$-algebra and $\mathrm{E}_{\mathrm{q}}$ coincides with the set of all hermitian minimal idempotents in $B$. Since $A$ is a left ideal of $B$, it is well-known that there exists a constant $k$ such that ||$b a|| \leq k|b| \quad|| a||$ for $a 11 \quad b$ in $B$ and $a$ in $A$. Then 
$\| P_{f A}(x)||=|| f x|| \leq k|f| \quad|| x||=k|| x||$ for all $x$ in $A$ and $f$ in $E_{q}$. Hence $\left\{P_{f A}: f \in E_{q}\right\}$ is bounded. It remains to show that it is closed. Let $\left\{\mathrm{P}_{f_{n} A}\right\}$ be a Cauchy sequence, where $f_{n} \in E_{q}$. We show that, for $m$ and $n$ large enough, $f_{m}$ and $f_{n}$ are contained in the same minimal closed twosided ideal. Suppose this is not so. Then there exists some minimal closed two-sided ideal $I_{\lambda_{n}}$ of $A$ such that $\mathrm{f}_{\mathrm{n}} \varepsilon \mathrm{I}_{\lambda_{\mathrm{n}}}$, but $\mathrm{f}_{\mathrm{m}} \notin \mathrm{I}_{\lambda_{\mathrm{n}}}$. Let $\mathrm{H}_{\lambda_{\mathrm{n}}}$ be the minimal left ideal in $I_{\lambda_{n}}$. Since $\left|f_{n}\right|=1$, we can choose $h_{n} \varepsilon H_{n}$ such that $\left|f_{n} h_{n}\right|>1 / 2$ with $\left|h_{n}\right|=1$. Since $f_{m} I_{\lambda_{n}}=(0)$, by (4.3) we have

$$
\begin{aligned}
1 / 2<\left|f_{n} h_{n}\right| & =\left|f_{n} h_{n}-f_{m} h_{n}\right| \leq M|| f_{n} h_{n}-f_{m} h_{n}|| \\
& \leq M|| P_{f_{n} A}-P_{f_{m} A}||\left|h_{n}\right|=M|| P_{f_{n} A}-P_{f_{m} A}|| .
\end{aligned}
$$

But $\left\{\mathrm{P}_{\mathrm{f}_{\mathrm{n}} \mathrm{A}}\right\}$ is a Cauchy sequence; a contradiction. Therefore, we can assume that $f_{m}$ and $f_{n}$ belong to the same $I_{\lambda_{n}}$. Hence,

$$
\begin{aligned}
\left|\mathrm{f}_{\mathrm{n}}-\mathrm{f}_{\mathrm{m}}\right| & =\sup \left\{\left|\left(\mathrm{f}_{\mathrm{n}}-\mathrm{f}_{\mathrm{m}}\right) \mathrm{h}\right|: \mathrm{h} \varepsilon \mathrm{H}_{\lambda_{\mathrm{n}}} \text { and }|\mathrm{h}| \leq 1\right\} \\
& \leq \mathrm{M}|| \mathrm{P}_{\mathrm{f}_{\mathrm{n}} \mathrm{A}}-\mathrm{P}_{\mathrm{f}_{\mathrm{m}} \mathrm{A}}||
\end{aligned}
$$

and so $\left\{\mathrm{f}_{\mathrm{n}}\right\}$ is a Cauchy sequence in $|\cdot|$. Since $\mathrm{E}_{\mathrm{q}}$ is closed in $|\cdot|$ by Theorem 4.2, in [11], $\mathrm{f}_{\mathrm{n}} \rightarrow \mathrm{f}$ in $|\cdot|$ for some $\mathrm{f}$ in $\mathrm{E}_{\mathrm{q}}$. Since

$$
\left\|\left(P_{f_{n} A}-P_{f A}\right)(x)\right\|=|| f_{n} x-f x|| \leq k\left|f_{n}-f\right| \quad|| x||
$$

for all $x$ in $A, P_{f_{n} A} \rightarrow P_{f A}$ and so $\left\{P_{f A}: f \varepsilon E_{q}\right\}$ is closed. This completes the proof. 


\section{REFERENCES}

1. Alexander, F. E. Representation Theorems for Complemented Algebras, Trans. Amer. Math. Soc. 148 (1970) 385-398.

2. Alexander, F. E. On Complemented and Annihilator Algebras, Glasgow J. Math. 10 (1969) 38-45.

3. Alexander, F. E. and B. J. Tomiuk Complemented B*-Algebras, Trans. Amer. Math. Soc. 137 (1969) 459-480.

4. Husain, T. and P. K. Wong Quasi-Complemented Algebras, Trans. Amer. Math. Soc. 174 (1972) 141-154.

5. Rickart, C. E. General Theory of Banach Algebras, The University Series in Higher Math., Van Nostrand, Princeton, New Jersey, 1960.

6. Tomiuk, B. J. Structure Theory of Complemented Banach Algebras, Canad. J. Math. 14 (1962) 651-659.

7. Wong, P. K. Continuous Complementors on B*-Algebras, Pacific J. Math. 33 (1970) 255-260.

8. Wong, P. K. On the Arens Products and Certain Banach Algebras, Trans. Amer. Math. Soc. 180 (1973) 437-448.

9. Wong, P. K. A Note on Annihilator and Complemented Banach Algebras, J. Australian Math. Soc. 18 (1974) 474-481.

10. Wong, P. K. A Minimax Formula for Dual B*-Algebras, Trans. Amer. Math. Soc. 224 (1976) 281-298.

11. Wong, P. K. Quasi-Complementors and Complementors on Certain Banach Algebras, Proc. Amer. Math. Soc. 65 (1977) 287-292. 


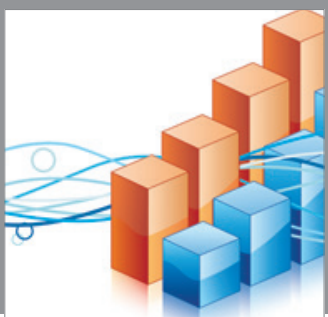

Advances in

Operations Research

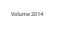



\section{The Scientific} World Journal


International Journal of

Mathematics and

Mathematical

Sciences
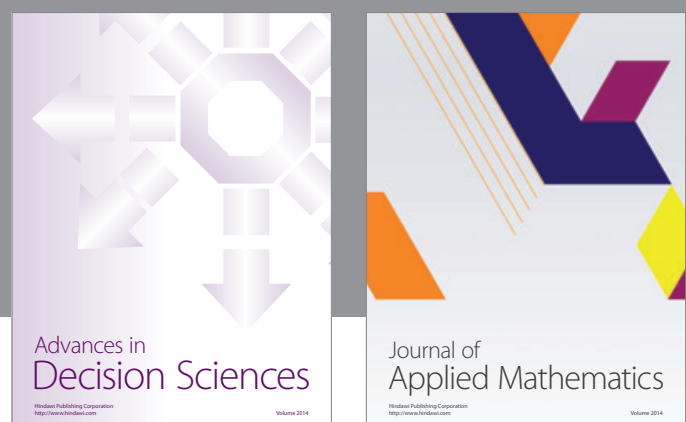

Journal of

Applied Mathematics
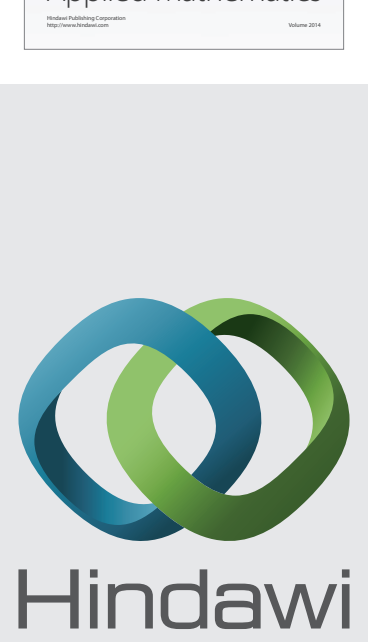

Submit your manuscripts at http://www.hindawi.com
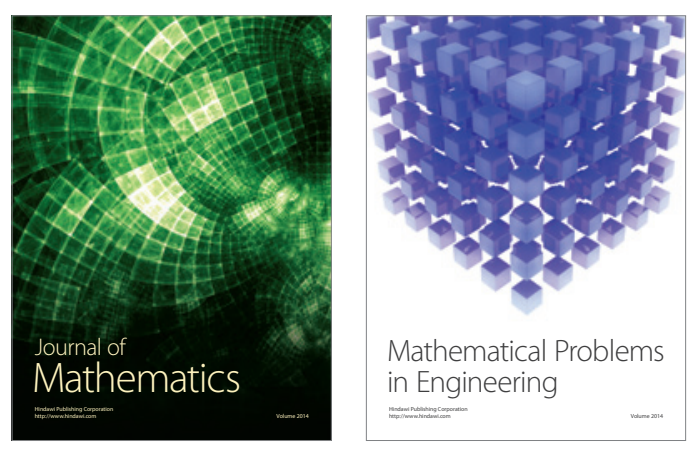

Mathematical Problems in Engineering
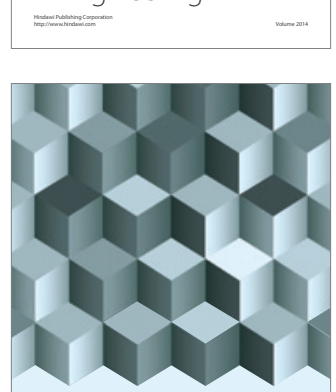

Journal of

Function Spaces
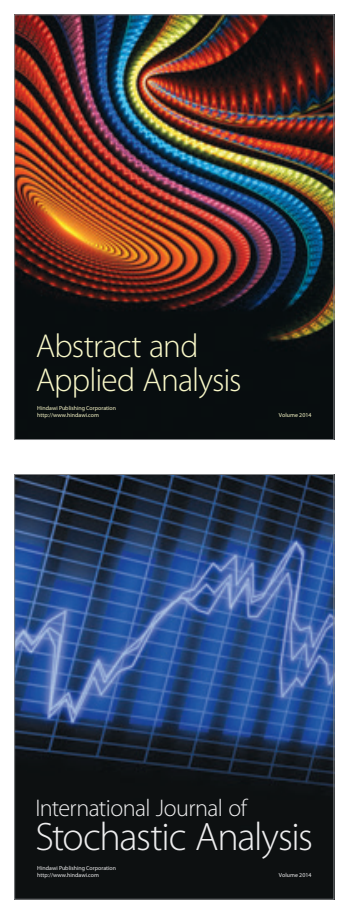

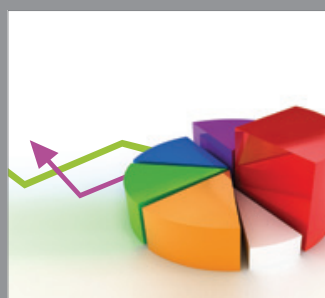

ournal of

Probability and Statistics

Promensencen
